\title{
Simplification of the Flux Function for a High-Order Gas-Kinetic Evolution Model
}

\author{
Guangzhao Zhou ${ }^{\mathrm{a}, *}, \mathrm{Kun} \mathrm{Xu}^{\mathrm{b}}$, Feng Liu ${ }^{\mathrm{c}}$ \\ ${ }^{a}$ College of Engineering, Peking University, Beijing 100871, China \\ ${ }^{b}$ Department of Mathematics, Hong Kong University of Science and Technology, Kowloon, Hong Kong, China \\ ${ }^{c}$ Department of Mechanical and Aerospace Engineering, University of California, Irvine, CA 92697-3975, United States
}

\begin{abstract}
High-order (higher-than 2nd-order) gas-kinetic schemes for solving the Navier-Stokes equations have been studied in recent years. In addition to the use of high-order reconstruction techniques, many terms are used in the Taylor expansion of the equilibrium and non-equilibrium gas distribution functions in the high-order gas kinetic flux function. Therefore, a large number of coefficients need to be determined in the calculation of the time evolution of the gas distribution function at cell interfaces. As a consequence, the high-order flux function takes much more computational time than that of a 2nd-order gas-kinetic scheme. This paper aims to simplify the evolution model by two steps. Firstly, the coefficients related to the 2nd-order spatial and temporal derivatives of a distribution function are redefined to reduce the computational cost. Secondly, based on the physical analysis, some terms can be removed without loss of accuracy. As a result, through the simplifications, the computational efficiency of the high-order scheme is increased significantly. In addition, a self-adaptive numerical viscosity is designed to minimize the necessary numerical dissipation. Several numerical examples are tested to demonstrate the accuracy and robustness of the current scheme.
\end{abstract}

Keywords: High-order scheme, Gas-kinetic scheme, Gas evolution model

\section{Introduction}

Most of the classical flow solvers are based on the Euler or Navier-Stokes equations. An exact or approximate Riemann solver is usually adopted for the inviscid flux. The viscous flux is treated separately from the inviscid part. However, the gas-kinetic scheme (GKS) [1, 2] for the computation of compressible flows proceeds from the microscopic dynamic process. The gas distribution function is introduced to follow the gas evolution from a general initial condition in both space and time. Then all macroscopic flow variables are expressed as moments of the distribution function. Since the non-equilibrium part of the distribution

\footnotetext{
* Corresponding author

Email addresses: zgz@pku.edu.cn (Guangzhao Zhou), makxu@ust.hk (Kun Xu), fliu@uci.edu (Feng Liu)
}

Preprint submitted to Journal of Computational Physics

March 10, 2017

(C) 2017. This manuscript version is made available under the Elsevier user license

http://www.elsevier.com/open-access/userlicense/1.0/ 
function corresponds to the viscous terms, the calculation of the inviscid and viscous fluxes are performed simultaneously.

With growing demand for accurate numerical solution and continuous increase of computational power, more attention has been devoted to the development of high-order schemes in recent years. By high order here one generally refers to higher-than 2nd-order schemes. These schemes include the (weighted) essentially non-oscillatory scheme (ENO/WENO) [3, 4, the discontinuous Galerkin method (DG) [5, 6], the compact nonlinear scheme [7, 8, and the spectral volume/difference method (SV/SD) 9, 10. For the majority of the current high-order schemes, the high-order accuracy is mostly associated with a high-order reconstruction. While the Riemann solver, which is responsible for the evolution process at the cell interface, stays the same as it is in low-order schemes. On the one hand, the Riemann solver is intrinsically one dimensional and cannot reflect the variation of variables in the second and third dimensions. Fluxes have to be evaluated at several Gaussian points on a cell interface to obtain accurate integration in the tangential direction(s) for 2-D and 3-D problems [11]. This will require disproportionally more computational cost in comparison with the 1-D case. On the other hand, the Riemann solver is a 1st-order description to the physical process and cannot reflect the variation of the status at the cell interface with time. To overcome this weakness, efforts have been taken to develop more accurate representations to the evolution process based on the generalized Riemann solver, e.g., the GRP scheme [12, 13] and the ADER method [14].

Recently, the original 2nd-order gas-kinetic scheme was extended to a higher order by several authors [15, 16, 17, 18. In addition to the use of high-order reconstruction, the evolution process also has a high-order property, which has been shown to be important in the construction of high-order schemes [19]. With the Taylor expansions in both the perpendicular and tangential directions of a cell interface, multidimensionality is achieved and no Gaussian points are theoretically needed. Moreover, the inclusion of the time-derivatives makes it more accurate than the Riemann solver.

The previous high-order GKS schemes have shown good performance for both inviscid and viscous flows 15, 16, 17, 18. However, since the evolution model is associated with a large number of space- and timedependent terms in the calculation of the flux, much more computational time is needed than that of a 2nd-order GKS. As reported in Ref. [19], with the same WENO reconstruction, the finite-volume 3rd-order GKS is 4 times slower than a finite-difference scheme with a Steger-Warming flux splitting method in the 2-D simulations. Therefore it is meaningful and necessary to reduce the computational cost of the current high-order GKS. This paper will follow the main idea in Ref. [17] for the construction of WENO-GKS. But, two simplifications are proposed to construct a more efficient scheme without loss of accuracy. The paper is organized as follows. In Section 2 , the general idea of the numerical method is introduced. Section 3 is a brief review of the previous high-order gas-kinetic evolution model as the baseline model. Section 4 shows 
the details of the simplifications on the baseline model. The accuracy and efficiency of the simplified method is validated in Section 5 by several standard test cases, followed by results of a few other test cases of interest in Section 6. Finally, conclusions are given in Section 7 .

\section{Numerical Procedure}

We present a brief introduction to the standard procedure of gas-kinetic schemes. More details can be found in Ref. [1, 2].

The BGK equation is [20:

$$
f_{t}+\boldsymbol{u} \cdot \nabla f=\frac{g-f}{\tau}
$$

where $f$ is the gas distribution function, $g$ is the equilibrium distribution that $f$ approaches, and $\boldsymbol{u}=(u, v)^{T}$ is the particle velocity. $\tau$ is defined as the collision time (time between collisions). It is related to the dynamic viscosity and pressure by $\tau=\mu / p$. The equilibrium function, known as the Maxwellian distribution, is

$$
g=\rho\left(\frac{\lambda}{\pi}\right)^{\frac{K+2}{2}} e^{-\lambda\left[(u-U)^{2}+(v-V)^{2}+\xi^{2}\right]}
$$

for 2-D flow, where $\rho$ is the density, $U, V$ are macroscopic velocities in $x$ and $y$ directions, respectively. $\lambda=m / 2 k T$, where $m$ is the molecular mass, $k$ is the Boltzmann constant and $T$ is the temperature. $K$ is the number of internal degrees of freedom which equals to 3 for diatomic molecules. $\xi$ is the internal variable with $\xi^{2}=\xi_{1}^{2}+\xi_{2}^{2}+\cdots+\xi_{K}^{2}$.

From Eqs. (1) and (2), it is clear that $f$ is a function of $\boldsymbol{x}, t, \boldsymbol{u}$ and $\xi$. The macroscopic variables $\rho$, $U, V$ and $T$ appear as coefficients that are local constants. The conservative variables are related to the distribution function by the following equation:

$$
\boldsymbol{W}=(\rho, \rho U, \rho V, \rho E)^{T}=\int g \boldsymbol{\psi} d \Xi,
$$

where $E$ is the total energy density.

Once the distribution function $f$ is obtained, the flux at a cell interface normal to the $x$ direction can be expressed as

$$
\boldsymbol{F}=\int u f \psi d \Xi
$$

where $d \Xi=d u d v d \xi, d \xi=d \xi_{1} d \xi_{2} \cdots d \xi_{K}$, and $\boldsymbol{\psi}$ is the vector of moments:

$$
\boldsymbol{\psi}=\left(\psi_{1}, \psi_{2}, \psi_{3}, \psi_{4}\right)^{T}=\left(1, u, v, \frac{u^{2}+v^{2}+\xi^{2}}{2}\right)^{T} .
$$

In addition, we have

$$
\int(g-f) \boldsymbol{\psi} d \Xi \equiv \mathbf{0}
$$


This is due to the conservation property of $\boldsymbol{W}$. It is valid for any $\boldsymbol{x}$ and $t$.

For a rectangular cell $\left[x_{i-1 / 2}, x_{i+1 / 2}\right] \times\left[y_{j-1 / 2}, y_{j+1 / 2}\right]$ with dimensions of $\Delta x_{i}=x_{i+1 / 2}-x_{i-1 / 2}$ and $\Delta y_{j}=y_{j+1 / 2}-y_{j-1 / 2}$, the cell-averaged conservative variable $\boldsymbol{W}_{i j}$ is updated from the time $t_{n}$ to $t_{n+1}$ as follows:

$$
\begin{aligned}
\boldsymbol{W}_{i j}^{n+1}=\boldsymbol{W}_{i j}^{n} & -\frac{1}{\Delta x_{i} \Delta y_{j}} \int_{t_{n}}^{t_{n+1}} \int_{-\frac{1}{2} \Delta y_{j}}^{\frac{1}{2} \Delta y_{j}}\left[\boldsymbol{F}_{i+1 / 2}(t, y)-\boldsymbol{F}_{i-1 / 2}(t, y)\right] d y d t \\
& -\frac{1}{\Delta x_{i} \Delta y_{j}} \int_{t_{n}}^{t_{n+1}} \int_{-\frac{1}{2} \Delta x_{i}}^{\frac{1}{2} \Delta x_{i}}\left[\boldsymbol{F}_{j+1 / 2}(t, x)-\boldsymbol{F}_{j-1 / 2}(t, x)\right] d x d t .
\end{aligned}
$$

The above equation is exact. In a conventional high-order finite-volume scheme, the surface and time integration on the RHS is replaced by Gaussian quadrature and a multi-step Runge-Kutta scheme of appropriate order, respectively. In the high-order GKS scheme, approximate functions of $\boldsymbol{F}$ are obtained by expanding $f$ in $x, y$ and $t$, and the surface and time integration is done analytically.

To get $f$, we use the analytical integral solution to Eq. (1):

$$
f(\boldsymbol{x}, t, \boldsymbol{u}, \xi)=\frac{1}{\tau} \int_{0}^{t} g\left(\boldsymbol{x}^{\prime}, t^{\prime}, \boldsymbol{u}, \xi\right) e^{-\left(t-t^{\prime}\right) / \tau} d t^{\prime}+e^{-t / \tau} f_{0}(\boldsymbol{x}-\boldsymbol{u} t, \boldsymbol{u}, \xi),
$$

where $\boldsymbol{x}^{\prime}=\boldsymbol{x}-\boldsymbol{u}\left(t-t^{\prime}\right)$ is the particle trajectory. Therefore, $f$ depends on the equilibrium distribution function $g$ and the initial distribution function $f_{0}$. The problem is then how to model these two functions.

We summarize the procedure in a time step:

(1) Reconstruction. Based on the current values of the averaged conservative variables, construct the values and their spatial derivatives at the midpoint of the cell interface on both sides. This could be done by various techniques. For this paper, the WENO method is applied.

(2) Modelling of evolution. Model $f_{0}$ and $g$ from the reconstructed conservative variables. Then get $f$ via Eq. (8). This paper aims to make this part more efficient.

(3) Flux integration. Obtain the flux at each cell interface according to Eq. (4).

(4) Update of conservative variables. This is done following Eq. (7).

The reconstruction part can be found in Ref. [17]. A standard 5th-order WENO-JS method [21] is applied in the direction perpendicular to the cell interface to determine the line-averaged values of the variables on both sides of the interface. Following the suggestion in Ref. [22, characteristic variables are used instead of the conservative variables. After that, a 3rd-order interpolation involving the nearby line-averaged values is performed in the tangential direction to obtain spatially distributed values along the interface. First and second-order derivatives in both $x$ and $y$ directions can be obtained from the reconstructed values and the cell-averaged values, which will be used in the evolution process. 


\section{The Baseline Gas-Kinetic Evolution Model}

In this section we introduce the model proposed in Ref. [17, as a baseline for further modifications. For simplicity, consider a cell interface normal to the $x$ axis centered at $(x, y)=(0,0)$.

Let $g$ denote the Maxwellian distribution function at the point $(x, y, t)=(0,0,0)$. Then $\tilde{g}$, the equilibrium distribution in the neighbourhood can be expressed via the Taylor expansion. To the second order in space and time, it is written as:

$$
\tilde{g}(\boldsymbol{x}, t, \boldsymbol{u}, \xi)=g+g_{x} x+g_{y} y+g_{t} t+\frac{1}{2} g_{x x} x^{2}+\frac{1}{2} g_{y y} y^{2}+\frac{1}{2} g_{t t} t^{2}+g_{x y} x y+g_{x t} x t+g_{y t} y t .
$$

Introducing the coefficients below:

$$
\begin{aligned}
& a_{1}=g_{x} / g, \quad a_{2}=g_{y} / g, \quad A=g_{t} / g \\
& d_{11}=\frac{\partial a_{1}}{\partial x}, \quad d_{12}=\frac{\partial a_{1}}{\partial y}=\frac{\partial a_{2}}{\partial x}, \quad d_{22}=\frac{\partial a_{2}}{\partial y} \\
& b_{1}=\frac{\partial a_{1}}{\partial t}=\frac{\partial A}{\partial x}, \quad b_{2}=\frac{\partial a_{2}}{\partial t}=\frac{\partial A}{\partial y}, \quad B=\frac{\partial A}{\partial t},
\end{aligned}
$$

Eq. (9) becomes

$$
\begin{aligned}
\tilde{g}(\boldsymbol{x}, t, \boldsymbol{u}, \xi)= & +g a_{1} x+g a_{2} y+g A t+\frac{1}{2} g\left(a_{1}^{2}+d_{11}\right) x^{2}+\frac{1}{2} g\left(a_{2}^{2}+d_{22}\right) y^{2}+\frac{1}{2} g\left(A^{2}+B\right) t^{2} \\
& +g\left(a_{1} a_{2}+d_{12}\right) x y+g\left(A a_{1}+b_{1}\right) x t+g\left(A a_{2}+b_{2}\right) y t .
\end{aligned}
$$

Consider the non-equilibrium distribution function $f$. According to the Chapman-Enskog expansion, to the order of the Navier-Stokes equations, $f$ and $g$ have the following relation [23]:

$$
f=g-\tau D g=g-\tau\left(g_{t}+u g_{x}+v g_{y}\right)
$$

Similarly, by applying the Taylor expansion for each term and neglecting high-order derivatives of $g$, we get

$$
\begin{aligned}
\tilde{f}(\boldsymbol{x}, t, \boldsymbol{u}, \xi)= & g+g_{x} x+g_{y} y+g_{t} t+\frac{1}{2} g_{x x} x^{2}+\frac{1}{2} g_{y y} y^{2}+\frac{1}{2} g_{t t} t^{2}+g_{x y} x y+g_{x t} x t+g_{y t} y t \\
& -\tau\left(g_{t}+g_{x t} x+g_{y t} y+g_{t t} t\right)-\tau u\left(g_{x}+g_{x x} x+g_{x y} y+g_{x t} t\right)-\tau v\left(g_{y}+g_{x y} x+g_{y y} y+g_{y t} t\right) .
\end{aligned}
$$

With the coefficients defined in Eq. [10, the expression of the non-equilibrium distribution valid in the neighbourhood of $(x, y, t)=(0,0,0)$ is:

$$
\begin{aligned}
\tilde{f}(\boldsymbol{x}, t, \boldsymbol{u}, \xi)= & g+g a_{1} x+g a_{2} y+g A t+\frac{1}{2} g\left(a_{1}^{2}+d_{11}\right) x^{2}+\frac{1}{2} g\left(a_{2}^{2}+d_{22}\right) y^{2}+\frac{1}{2} g\left(A^{2}+B\right) t^{2} \\
& +g\left(a_{1} a_{2}+d_{12}\right) x y+g\left(A a_{1}+b_{1}\right) x t+g\left(A a_{2}+b_{2}\right) y t \\
& -\tau\left[g A+g\left(A a_{1}+b_{1}\right) x+g\left(A a_{2}+b_{2}\right) y+g\left(A^{2}+B\right) t\right] \\
& -\tau u\left[g a_{1}+g\left(a_{1}^{2}+d_{11}\right) x+g\left(a_{1} a_{2}+d_{12}\right) y+g\left(A a_{1}+b_{1}\right) t\right] \\
& -\tau v\left[g a_{2}+g\left(a_{1} a_{2}+d_{12}\right) x+g\left(a_{2}^{2}+d_{22}\right) y+g\left(A a_{2}+b_{2}\right) t\right] .
\end{aligned}
$$


Note that for an arbitrarily-given equilibrium state $g$, there exist $\tilde{g}$ and $\tilde{f}$ corresponding to $g$. Then we have the form $\tilde{g}=\tilde{g}(g, \boldsymbol{x}, t, \boldsymbol{u}), \tilde{f}=\tilde{f}(g, \boldsymbol{x}, t, \boldsymbol{u})$ ( $\xi$ is indirectly related to $\tilde{g}$ and $\tilde{f}$ via $g$ ). Now model the unknown functions in the solution (8) as the following.

The initial state at the cell interface should be discontinuous:

$$
f_{0}(\boldsymbol{x}, \boldsymbol{u}, \xi)= \begin{cases}f_{0}^{l}(\boldsymbol{x}, \boldsymbol{u}, \xi)=\tilde{f}^{l}\left(g_{0}^{l}, \boldsymbol{x}, 0, \boldsymbol{u}\right), & x \leq 0, \\ f_{0}^{r}(\boldsymbol{x}, \boldsymbol{u}, \xi)=\tilde{f}^{r}\left(g_{0}^{r}, \boldsymbol{x}, 0, \boldsymbol{u}\right), & x>0\end{cases}
$$

where $g_{0}^{l}$ and $g_{0}^{r}$ correspond to the reconstructed conservative variables at the left and right sides of the cell interface, respectively. i.e.,

$$
\boldsymbol{W}^{l}=\int g_{0}^{l} \boldsymbol{\psi} d \Xi, \quad \boldsymbol{W}^{r}=\int g_{0}^{r} \boldsymbol{\psi} d \Xi .
$$

The equilibrium distribution function in the integral solution (8) is replaced by

$$
g(\boldsymbol{x}, t, \boldsymbol{u}, \xi)=\tilde{g}\left(g^{e}, \boldsymbol{x}, t, \boldsymbol{u}\right)
$$

where $g^{e}$ is formed from the colliding particles from both sides of the interface. According to the relation (3), it is obtained by:

$$
\int g^{e} \boldsymbol{\psi} d \Xi=\boldsymbol{W}^{e}=\int_{u \geq 0} g_{0}^{l} \boldsymbol{\psi} d \Xi+\int_{u<0} g_{0}^{r} \boldsymbol{\psi} d \Xi .
$$

By replacing $f_{0}^{l, r}$ and $g$ with $\tilde{f}^{l, r}$ and $\tilde{g}$, we get an approximation to the exact distribution function $f$ via Eq. (8). The accuracy of the approximation is related to the order of the Taylor expansions for $\tilde{f}^{l, r}$ and $\tilde{g}$. A 1st-order expansion is enough in the 2nd-order gas-kinetic scheme [2]. For the current high-order scheme, we employ the 2nd-order expansion to more accurately reflect the variation of the distribution functions with $x$, $y$ and $t$.

The coefficients $a_{1}, a_{2}, A, \cdots$ in the distribution functions (11) and 14 are determined by the conservative variables. Each coefficient can be written as $\Lambda=\Lambda_{1} \psi_{1}+\Lambda_{2} \psi_{2}+\Lambda_{3} \psi_{3}+\Lambda_{4} \psi_{4}$. With the help of the relation in Eq. (6) and the definition

$$
\langle\cdots\rangle=\int g(\cdots) \psi d \Xi
$$

they can be derived as follows:

$$
\begin{aligned}
& \left\langle a_{1}\right\rangle=\boldsymbol{W}_{x} \rightarrow a_{1}, \quad\left\langle a_{2}\right\rangle=\boldsymbol{W}_{y} \rightarrow a_{2}, \quad\left\langle a_{1} u+a_{2} v+A\right\rangle=\mathbf{0} \rightarrow A, \\
& \left\langle a_{1}^{2}+d_{11}\right\rangle=\boldsymbol{W}_{x x} \rightarrow d_{11}, \quad\left\langle a_{2}^{2}+d_{22}\right\rangle=\boldsymbol{W}_{y y} \rightarrow d_{22}, \quad\left\langle a_{1} a_{2}+d_{12}\right\rangle=\boldsymbol{W}_{x y} \rightarrow d_{12}, \\
& \left\langle\left(a_{1}^{2}+d_{11}\right) u+\left(a_{1} a_{2}+d_{12}\right) v+A a_{1}+b_{1}\right\rangle=\mathbf{0} \rightarrow b_{1}, \\
& \left\langle\left(a_{1} a_{2}+d_{12}\right) u+\left(a_{2}^{2}+d_{22}\right) v+A a_{2}+b_{2}\right\rangle=\mathbf{0} \rightarrow b_{2}, \\
& \left\langle\left(A a_{1}+b_{1}\right) u+\left(A a_{2}+b_{2}\right) v+A^{2}+B\right\rangle=\mathbf{0} \rightarrow B .
\end{aligned}
$$


All moments can be calculated explicitly. Details can be found in Ref. [2].

With all the above preparations, the final expression of $f$ at $x=0$ is written as (see Ref. [17):

$$
\begin{aligned}
f(0, y, t, \boldsymbol{u}, \xi)= & \frac{1}{\tau} \int_{0}^{t} g\left(-u\left(t-t^{\prime}\right), y-v\left(t-t^{\prime}\right), t^{\prime}, \boldsymbol{u}, \xi\right) e^{-\left(t-t^{\prime}\right) / \tau} d t^{\prime} \\
& +e^{-t / \tau} f_{0}(-u t, y-v t, \boldsymbol{u}, \xi) .
\end{aligned}
$$

The first term on the RHS of Eq. 21] is

$$
\begin{aligned}
& \frac{1}{\tau} \int_{0}^{t} g\left(-u\left(t-t^{\prime}\right), y-v\left(t-t^{\prime}\right), t^{\prime}, \boldsymbol{u}, \xi\right) e^{-\left(t-t^{\prime}\right) / \tau} d t^{\prime} \\
= & C_{1} g^{e}+C_{2} g^{e} a_{1}^{e} u+C_{1} g^{e} a_{2}^{e} y+C_{2} g^{e} a_{2}^{e} v+C_{3} g^{e} A^{e}+\frac{1}{2} C_{4} g^{e}\left(\left(a_{1}^{e}\right)^{2}+d_{11}^{e}\right) u^{2}+\frac{1}{2} C_{1} g^{e}\left(\left(a_{2}^{e}\right)^{2}+d_{22}\right) y^{2} \\
& +C_{2} g^{e}\left(\left(a_{2}^{e}\right)^{2}+d_{22}^{e}\right) v y+\frac{1}{2} C_{4} g^{e}\left(\left(a_{2}^{e}\right)^{2}+d_{22}^{e}\right) v^{2}+C_{2} g^{e}\left(a_{1}^{e} a_{2}^{e}+d_{12}^{e}\right) u y+C_{4} g^{e}\left(a_{1}^{e} a_{2}^{e}+d_{12}^{e}\right) u v \\
& +\frac{1}{2} C_{5} g^{e}\left(\left(A^{e}\right)^{2}+B^{e}\right)+C_{6} g^{e}\left(A^{e} a_{1}^{e}+b_{1}^{e}\right) u+C_{3} g^{e}\left(A^{e} a_{2}^{e}+b_{2}^{e}\right) y+C_{6} g^{e}\left(A^{e} a_{2}^{e}+b_{2}^{e}\right) v .
\end{aligned}
$$

The second term is

$$
e^{-t / \tau} f_{0}(-u t, y-v t, \boldsymbol{u}, \xi)= \begin{cases}e^{-t / \tau} f_{0}^{l}(-u t, y-v t, \boldsymbol{u}, \xi), & u \geq 0, \\ e^{-t / \tau} f_{0}^{r}(-u t, y-v t, \boldsymbol{u}, \xi), & u<0\end{cases}
$$

where

$$
\begin{aligned}
& e^{-t / \tau} f_{0}^{l, r}(-u t, y-v t, \boldsymbol{u}, \xi) \\
= & C_{7} g_{0}^{l, r}\left[1-\tau\left(a_{1}^{l, r} u+a_{2}^{l, r} v+A^{l, r}\right)\right] \\
& +C_{8} g_{0}^{l, r}\left[a_{1}^{l, r} u-\tau\left(\left(\left(a_{1}^{l, r}\right)^{2}+d_{11}^{l, r}\right) u^{2}+\left(a_{1}^{l, r} a_{2}^{l, r}+d_{12}^{l, r}\right) u v+\left(A^{l, r} a_{1}^{l, r}+b_{1}^{l, r}\right) u\right)\right] \\
& +C_{7} g_{0}^{l, r}\left[a_{2}^{l, r}-\tau\left(\left(a_{1}^{l, r} a_{2}^{l, r}+d_{12}^{l, r}\right) u+\left(\left(a_{2}^{l, r}\right)^{2}+d_{22}^{l, r}\right) v+A^{l, r} a_{2}^{l, r}+b_{2}^{l, r}\right)\right] y \\
& +C_{8} g_{0}^{l, r}\left[a_{2}^{l, r} v-\tau\left(\left(a_{1}^{l, r} a_{2}^{l, r}+d_{12}^{l, r}\right) u v+\left(\left(a_{2}^{l, r}\right)^{2}+d_{22}^{l, r}\right) v^{2}+\left(A^{l, r} a_{2}^{l, r}+b_{2}^{l, r}\right) v\right)\right] \\
& +\frac{1}{2} C_{9} g_{0}^{l, r}\left(\left(a_{1}^{l, r}\right)^{2}+d_{11}^{l, r}\right) u^{2}+\frac{1}{2} C_{7} g_{0}^{l, r}\left(\left(a_{2}^{l, r}\right)^{2}+d_{22}^{l, r}\right) y^{2}+C_{8} g_{0}^{l, r}\left(\left(a_{2}^{l, r}\right)^{2}+d_{22}^{l, r}\right) v y \\
& +\frac{1}{2} C_{9} g_{0}^{l, r}\left(\left(a_{2}^{l, r}\right)^{2}+d_{22}^{l, r}\right) v^{2}+C_{8} g_{0}^{l, r}\left(a_{1}^{l, r} a_{2}^{l, r}+d_{12}^{l, r}\right) u y+C_{9} g_{0}^{l, r}\left(a_{1}^{l, r} a_{2}^{l, r}+d_{12}^{l, r}\right) u v .
\end{aligned}
$$

The coefficients in the above equations are

$$
\begin{aligned}
& C_{1}=1-e^{-t / \tau_{n}}, \quad C_{2}=(t+\tau) e^{-t / \tau_{n}}-\tau, \quad C_{3}=t-\tau+\tau e^{-t / \tau_{n}}, \\
& C_{4}=\left(-t^{2}-2 \tau t\right) e^{-t / \tau_{n}}, \quad C_{5}=t^{2}-2 \tau t, \quad C_{6}=-\tau t\left(1+e^{-t / \tau_{n}}\right), \\
& C_{7}=e^{-t / \tau_{n}}, \quad C_{8}=-t e^{-t / \tau_{n}}, \quad C_{9}=t^{2} e^{-t / \tau_{n}},
\end{aligned}
$$

where $\tau_{n}$ is the numerical collision time which contains both physical and numerical dissipation [17. 


\section{Modifications and Discussions on the Baseline Evolution Model}

\subsection{Simplification 1}

The first simplification is to rearrange the coefficients in Eq. (10). In the baseline model, all 2nd-order derivatives of the Maxwellian distribution function are expressed by a combination of three coefficients. For example, $g_{x x} / g=a_{1}^{2}+d_{11}, g_{x t} / g=A a_{1}+b_{1}$. We observe from Eqs. 222 and 24 that the same combinations always appear during the calculation. It is thus convenient to replace each combination by a single coefficient. A new set of coefficients is introduced instead of those in Eq. 10p:

$$
\begin{aligned}
& a_{x}=g_{x} / g, \quad a_{y}=g_{y} / g, \quad a_{t}=g_{t} / g, \\
& a_{x x}=g_{x x} / g, \quad a_{y y}=g_{y y} / g, \quad a_{x y}=g_{x y} / g, \\
& a_{x t}=g_{x t} / g, \quad a_{y t}=g_{y t} / g, \quad a_{t t}=g_{t t} / g .
\end{aligned}
$$

Eq. (20) is then replaced by

$$
\begin{aligned}
& \left\langle a_{x}\right\rangle=\boldsymbol{W}_{x} \rightarrow a_{x}, \quad\left\langle a_{y}\right\rangle=\boldsymbol{W}_{y} \rightarrow a_{y}, \quad\left\langle a_{x} u+a_{y} v+a_{t}\right\rangle=\mathbf{0} \rightarrow a_{t}, \\
& \left\langle a_{x x}\right\rangle=\boldsymbol{W}_{x x} \rightarrow a_{x x}, \quad\left\langle a_{y y}\right\rangle=\boldsymbol{W}_{y y} \rightarrow a_{y y}, \quad\left\langle a_{x y}\right\rangle=\boldsymbol{W}_{x y} \rightarrow a_{x y}, \\
& \left\langle a_{x x} u+a_{x y} v+a_{x t}\right\rangle=\mathbf{0} \rightarrow a_{x t}, \quad\left\langle a_{x y} u+a_{y y} v+a_{y t}\right\rangle=\mathbf{0} \rightarrow a_{y t}, \\
& \left\langle a_{x t} u+a_{y t} v+a_{t t}\right\rangle=\mathbf{0} \rightarrow a_{t t} .
\end{aligned}
$$

The above modifications have the following properties:

(1) The new definition does not change the total number of coefficients, so the number of degrees of freedom remains the same. Therefore, the macroscopic information used for the evolution process is not reduced, eliminating the risk of loss of accuracy.

(2) Both the old set of coefficients defined by Eq. 10 and the new set defined by Eq. 26) are represented as a linear (first-order) combination of $\psi_{1}, \psi_{2}, \psi_{3}$ and $\psi_{4}$ to fully recover all first- and second-order derivatives of the macroscopic conservative flow variables in space and time by taking the moments of the resulting distribution function. The original set of coefficients leads to additional higher-order terms of the particle velocity in the expansion, which may contribute in the integral solution of the gas-kinetic scheme slight differences in the flux calculation. But such differences are beyond the Chapman-Enskog expansion. In fact, computations show that avoidance of such additional higher-order expansion terms by the new set of coefficients improves the computational accuracy (see Section 5.4 .

(3) Clearly the computational cost of determining the coefficients in Eq. 27. is much less compared to that of determining those in Eq. 20. 
(4) The solution for $f$ is much simplified with the new coefficients. The combinations of coefficients in Eqs. 22 and 24 are replaced by single coefficients as follows:

$$
\begin{aligned}
& a_{1}^{2}+d_{11} \rightarrow a_{x x}, \quad a_{2}^{2}+d_{22} \rightarrow a_{y y}, \quad a_{1} a_{2}+d_{12} \rightarrow a_{x y}, \\
& A a_{1}+b_{1} \rightarrow a_{x t}, \quad A a_{2}+b_{2} \rightarrow a_{y t}, \quad A^{2}+B \rightarrow a_{t t} .
\end{aligned}
$$

Notice that each coefficient is a summation of 4 terms, e.g.,

$$
a_{t t}=a_{t t}^{(1)}+a_{t t}^{(2)} u+a_{t t}^{(3)} v+a_{t t}^{(4)} \frac{u^{2}+v^{2}+\xi^{2}}{2},
$$

as a comparison, $A^{2}+B$ is expanded as

$$
\left(A^{(1)}+A^{(2)} u+A^{(3)} v+A^{(4)} \frac{u^{2}+v^{2}+\xi^{2}}{2}\right)^{2}+\left(B^{(1)}+B^{(2)} u+B^{(3)} v+B^{(4)} \frac{u^{2}+v^{2}+\xi^{2}}{2}\right),
$$

which contains many more terms than 4 . So the time saved is considerable.

(5) The original definition of coefficients leads to high-order terms of $\boldsymbol{\psi}$ in the calculation of the flux. In the expansion of $A^{2}+B$, the highest power on $u, v$ and $\xi$ is 4 . While in the expansion of $a_{t t}$, the highest power is 2 . Without involving very high moments of the distribution function, the simplified scheme tends to be more stable.

\subsection{Simplification 2}

By substituting the coefficients and rearranging the terms, the final solution of the distribution function $f$ at the cell interface (Eqs. 21) - 25) can be expressed as following,

$$
\begin{aligned}
& f(0, y, t, \boldsymbol{u}, \xi) \\
& =\frac{1}{\tau} \int_{0}^{t} g\left(-u\left(t-t^{\prime}\right), y-v\left(t-t^{\prime}\right), t^{\prime}, \boldsymbol{u}, \xi\right) e^{-\left(t-t^{\prime}\right) / \tau} d t^{\prime}+e^{-t / \tau} f_{0}(-u t, y-v t, \boldsymbol{u}, \xi) \\
& =g^{e}+\frac{1}{2} g_{y y}^{e} y^{2}+g_{t}^{e} t+\frac{1}{2} g_{t t}^{e} t^{2}-\tau\left[\left(g_{t}^{e}+u g_{x}^{e}+v g_{y}^{e}\right)+\left(g_{t t}^{e}+u g_{x t}^{e}+v g_{y t}^{e}\right) t\right] \\
& -e^{-t / \tau}\left[\begin{array}{c}
g^{e}+\frac{1}{2} g_{y y}^{e} y^{2}-\left(u g_{x}^{e}+v g_{y}^{e}\right) t+\frac{1}{2}\left(u^{2} g_{x x}^{e}+2 u v g_{x y}^{e}+v^{2} g_{y y}^{e}\right) t^{2} \\
-\tau\left[\left(g_{t}^{e}+u g_{x}^{e}+v g_{y}^{e}\right)-\left(u g_{x t}^{e}+v g_{y t}^{e}+u^{2} g_{x x}^{e}+2 u v g_{x y}^{e}+v^{2} g_{y y}^{e}\right) t\right]
\end{array}\right] \\
& +e^{-t / \tau}\left\{\begin{array}{rlrl}
g^{l} & +\frac{1}{2} g_{y y}^{l} y^{2}-\left(u g_{x}^{l}+v g_{y}^{l}\right) t+\frac{1}{2}\left(u^{2} g_{x x}^{l}+2 u v g_{x y}^{l}+v^{2} g_{y y}^{l}\right) t^{2} & \\
& -\tau\left[\left(g_{t}^{l}+u g_{x}^{l}+v g_{y}^{l}\right)-\left(u g_{x t}^{l}+v g_{y t}^{l}+u^{2} g_{x x}^{l}+2 u v g_{x y}^{l}+v^{2} g_{y y}^{l}\right) t\right], \quad u \geq 0, \\
g^{r} & +\frac{1}{2} g_{y y}^{r} y^{2}-\left(u g_{x}^{r}+v g_{y}^{r}\right) t+\frac{1}{2}\left(u^{2} g_{x x}^{r}+2 u v g_{x y}^{r}+v^{2} g_{y y}^{r}\right) t^{2} & \\
& -\tau\left[\left(g_{t}^{r}+u g_{x}^{r}+v g_{y}^{r}\right)-\left(u g_{x t}^{r}+v g_{y t}^{r}+u^{2} g_{x x}^{r}+2 u v g_{x y}^{r}+v^{2} g_{y y}^{r}\right) t\right], \quad & & u<0 .
\end{array}\right.
\end{aligned}
$$

$+Y$. 
Here the terms with the factor $y$ is collected into $Y$, i.e., $Y=y H$, where $H$ is independent of $y$. Since the distribution function is to be integrated in the interval $\left[-\frac{1}{2} \Delta y, \frac{1}{2} \Delta y\right]$, these terms will vanish. So it is unnecessary to include them. Note that the expression Eq. (31) is the original physical one, we should keep in mind that all $e^{-t / \tau}$ is actually $e^{-t / \tau_{n}}$ in practical computations.

Consider the expression of $f$, the terms without the factor $e^{-t / \tau}$ are exactly a combination of the Chapman-Enskog expansion and the Taylor expansion based on $g^{e}$ at $(x, y, t)=(0,0,0)$. While the terms with the factor $e^{-t / \tau}$ have the same form for the initial discontinuous (left and right) state and the equilibrium state. But the sign in front of them is different. Therefore $f$ can be written formally as

$$
f=\widetilde{g^{e}}-\tau \widetilde{D g^{e}}+e^{-t / \tau}\left(E^{l, r}-E^{e}\right) .
$$

(1) In Euler cases, $\tau=\mu / p=0$ and the exponential part vanishes.

(2) In viscous cases, when the grid for computation is fine enough, the flow field is locally smooth. Hence the variables and their gradients on the left and right of the interface and the equilibrium state are identical: $E^{l, r}=E^{e}$. Then the exponential part vanishes.

(3) The exponential part makes sense only when there is a discontinuity at the cell interface.

Since for viscous flows an absolute discontinuity does not actually exist in the real world, the flow field is smooth everywhere if fine grids are adopted, in which case the exponential part disappears. Then it can be concluded that the dominating part of the distribution function is $\widetilde{g^{e}}-\tau \widetilde{D g^{e}}$, while $e^{-t / \tau}\left(E^{l, r}-E^{e}\right)$ plays a role of numerical dissipation, which is related to the relative scale of the computational grid and the physical structure thickness. In other words, the function of these terms is to suppress spurious numerical oscillations near discontinuities on coarse grids. Hence, they can be simplified appropriately.

From another aspect, it is well known that almost all reconstruction techniques including WENO encounter an order-reduction at discontinuities, and non-physical artificial viscosity is generally needed at such regions. Remember that the term $e^{-t / \tau_{n}}$ is practically used in place of $e^{-t / \tau}$. There is not a unique theory for the construction of $\tau_{n}$. These uncertainties make it meaningless to overly seek accurate values for the terms in the bracket behind $e^{-t / \tau_{n}}$. Then it is reasonable to just keep the primary terms in $E^{l, r}-E^{e}$ for providing necessary numerical dissipation.

Notice that $E^{e, l, r}$ contains both inviscid and viscous (which are related to $\tau$ ) terms. Since the viscosity generally plays the role of smoothing the flow field, the physical viscous effect is not dominant wherever a discontinuity exists. Then it is reasonable to get rid of all terms with the factor $\tau$. On the other hand, we can neglect all 2nd-order terms associated with small parameters $y$ and $t$, which are of the order $\Delta y$ and $\Delta t$, respectively. The resultant expression is

$$
E^{e, l, r}=g^{e, l, r}-\left(u g_{x}^{e, l, r}+v g_{y}^{e, l, r}\right) t .
$$


Our numerical results show that the 1st-order terms of $t$ are necessary. Thus this form cannot be further simplified. From various test cases, the differences between the results obtained with Eq. (33) and those obtained with the original form in Eq. (31) are very small. Neither of the two forms is found superior to the other in terms of accuracy and grid convergence.

We conclude by proposing the following final expression for the distribution function

$$
\begin{aligned}
f(0, y, t, \boldsymbol{u}, \xi)= & g^{e}+\frac{1}{2} g_{y y}^{e} y^{2}+g_{t}^{e} t+\frac{1}{2} g_{t t}^{e} t^{2}-\tau\left[\left(g_{t}^{e}+u g_{x}^{e}+v g_{y}^{e}\right)+\left(g_{t t}^{e}+u g_{x t}^{e}+v g_{y t}^{e}\right) t\right] \\
& -e^{-t / \tau}\left[g^{e}-\left(u g_{x}^{e}+v g_{y}^{e}\right) t\right]+e^{-t / \tau}\left\{\begin{array}{ll}
g^{l}-\left(u g_{x}^{l}+v g_{y}^{l}\right) t, & u \geq 0 \\
g^{r}-\left(u g_{x}^{r}+v g_{y}^{r}\right) t, & u<0
\end{array}\right\}+Y,
\end{aligned}
$$

which is obviously much simpler than the original Eq. (31). In the following sections we demonstrate that the simplified method has a good performance by several different kinds of test cases.

\subsection{The numerical collision time}

For Navier-Stokes solutions, the numerical collision time $\tau_{n}$ is also modified. In Ref. [17] the formula is written as:

$$
\tau=\frac{\mu}{p^{e}}, \quad \tau_{n}=\tau+\beta \Delta x \sqrt{\lambda^{e}}\left|\frac{p^{l}-p^{r}}{p^{l}+p^{r}}\right|
$$

where $\mu$ is the dynamic viscosity at the cell interface and $p^{e}$ and $\lambda^{e}$ take values corresponding to the equilibrium state. $\tau_{n}$ contains both physical and numerical dissipation. We find that since the scheme itself has the mechanism to suppress oscillations near discontinuities, extra artificial viscosity is needed only for flows with severe discontinuity. Then the following formula is designed:

$$
\tau_{n}=\tau+\alpha \Delta t e^{1-\eta^{-10}}, \quad \eta=\left|\frac{p^{l}-p^{r}}{p^{l}+p^{r}}\right| .
$$

where $\alpha$ is a constant and is taken to be 0.3 in our computations.

For 1-D Euler cases, setting $\tau=\tau_{n}=0$ does not cause any problem. For 2-D Euler cases, some numerical dissipation is needed. The physical collision time is still $\tau=0$. For the numerical collision time $\tau_{n}$, we use the formula in Ref. [18, which reads

$$
\tau_{n}=C_{1} \Delta t+C_{2} \Delta t\left|\frac{p^{l}-p^{r}}{p^{l}+p^{r}}\right|
$$

where $C_{1}=0.1$ and $C_{2}=1.0$.

\section{Validation of Accuracy and Efficiency}

The above proposed simplified scheme has been put to test on a wide range of test cases to demonstrate its computational efficiency and robustness while maintaining the same order of accuracy of the original high- 
order GKS scheme in Ref. [17. In this section, we perform quantitative studies of accuracy and efficiency of the simplified scheme by computing a few standard test cases.

The first two test cases involve 1-D and 2-D advection of smooth flows for which analytical solutions are available and the asymptotic order of accuracy of a numerical scheme may be definitively determined by a successive grid refinement study. These are also the same accuracy test cases used for the original method in Ref. 17. Two more test cases involving more complex inviscid and viscous flows with shock and contact discontinuities are computed to access the accuracy and quantify the speedup of the proposed method.

For cases where the flow fields are smooth, the derivatives of the macroscopic variables at the equilibrium state are obtained by a 5th-order interpolation, exactly the same as those in Ref. [17. In other cases where discontinuities may exist, the derivatives are calculated by a 3rd-order interpolation, which proved to be more robust for both the original and the simplified methods.

\subsection{Advection of density perturbation}

This is a 1-D problem. The initial condition is given by $\rho(x)=1+0.2 \sin (\pi x), U(x)=1$ and $p(x)=1$. The density perturbation propagates periodically in the computational domain $x \in[0,2]$. No artificial viscosity is added. In the first test for this case, a spatial grid refinement study is conducted with the fixed CFL number of 0.1. The computed density distribution at $t=2$ is compared with the analytical solution. Table 1 shows the $L_{\infty}, L_{1}$, and $L_{2}$ errors on the successively refined grids and the corresponding order of accuracy deduced from these errors. The result demonstrates the same 5th-order accuracy in space as those reported of the original scheme in Ref. [17. Subsequently, a time accuracy study is performed by varying the CFL number from 0.2 to 0.8 on the same grid with 800 cells. The results are listed in Table 2 which yield again the same 3rd-order accuracy as the original high-order GKS scheme in Ref. [17. Comparison of the results listed in Table 1 and 2 and those reported in Ref. [17 shows that the present method preserves not only the same asymptotic order of accuracy but also the same level of absolute errors as the original high-order GKS method.

\subsection{Isentropic periodic vortex propagation}

This is a 2 -D inviscid case, the computational domain is $[0,10] \times[0,10]$ with periodic boundary conditions in both directions. The initial condition is

$$
\begin{aligned}
& (U(x, y), V(x, y))=(1,1)+\frac{\kappa}{2 \pi} e^{0.5\left(1-r^{2}\right)}(-\bar{y}, \bar{x}), \\
& T(x, y)=1-\frac{(\gamma-1) \kappa^{2}}{8 \gamma \pi^{2}} e^{1-r^{2}}, \quad \rho=T^{1 /(\gamma-1)}, \quad p=\rho^{\gamma},
\end{aligned}
$$

where $r^{2}=\bar{x}^{2}+\bar{y}^{2}$ and $(\bar{x}, \bar{y})=(x-5, y-5)$. The vortex strength is $\kappa=5$. The density distribution at $t=10$ (after one period) is compared with the analytical solution which is identical to the initial state. 


\begin{tabular}{ccccccc}
\hline Grid & $L_{\infty}$ error & Order & $L_{1}$ error & Order & $L_{2}$ error & Order \\
\hline 400 & $1.2512 \times 10^{-10}$ & 5.0152 & $7.3580 \times 10^{-11}$ & 4.9265 & $8.1877 \times 10^{-11}$ & 4.9418 \\
200 & $4.0463 \times 10^{-9}$ & 5.1824 & $2.2376 \times 10^{-9}$ & 4.9844 & $2.5164 \times 10^{-9}$ & 4.9980 \\
100 & $1.4693 \times 10^{-7}$ & 5.0619 & $7.0834 \times 10^{-8}$ & 4.9999 & $8.0415 \times 10^{-8}$ & 5.0412 \\
50 & $4.9079 \times 10^{-6}$ & 4.8433 & $2.2665 \times 10^{-6}$ & 5.0473 & $2.6478 \times 10^{-6}$ & 5.0452 \\
20 & $4.1518 \times 10^{-4}$ & 4.3057 & $2.3115 \times 10^{-4}$ & 4.3690 & $2.6952 \times 10^{-4}$ & 4.3169 \\
10 & $8.2107 \times 10^{-3}$ & - & $4.7763 \times 10^{-3}$ & - & $5.3717 \times 10^{-3}$ & - \\
\hline
\end{tabular}

Table 1: Space accuracy test for the advection of density perturbation by the simplified scheme.

\begin{tabular}{ccccccc}
\hline CFL & $L_{\infty}$ error & Order & $L_{1}$ error & Order & $L_{2}$ error & Order \\
\hline 0.2 & $1.733 \times 10^{-11}$ & 2.907 & $1.190 \times 10^{-11}$ & 2.819 & $1.306 \times 10^{-11}$ & 2.834 \\
0.4 & $1.300 \times 10^{-10}$ & 2.994 & $8.398 \times 10^{-11}$ & 2.967 & $9.312 \times 10^{-11}$ & 2.970 \\
0.6 & $4.376 \times 10^{-10}$ & 2.996 & $2.797 \times 10^{-10}$ & 2.989 & $3.105 \times 10^{-10}$ & 2.990 \\
0.8 & $1.036 \times 10^{-9}$ & - & $6.609 \times 10^{-10}$ & - & $7.339 \times 10^{-10}$ & - \\
\hline
\end{tabular}

Table 2: Time accuracy test for the advection of density perturbation by the simplified scheme.

The CFL number, which was not mentioned in Ref. [17, is taken to be 0.2. Notice that although we use a 5th-order reconstruction in the direction perpendicular to the cell interfaces, the reconstruction used in the tangential direction is only 3rd order. Moreover, the present multidimensional evolution model is a 3rd-order one. Therefore, unlike in the above 1-D case where 5th-order spatial accuracy is achieved, 3rd-order accuracy is expected for the original and the simplified schemes for 2-D problems. The errors and corresponding convergence orders are listed in Table 3 A 3rd-order accuracy is observed, which is consistent with the conclusion in Ref. [17].

\begin{tabular}{ccccccc}
\hline Grid & $L_{\infty}$ error & Order & $L_{1}$ error & Order & $L_{2}$ error & Order \\
\hline 160 & $6.7176 \times 10^{-5}$ & 3.9933 & $8.9787 \times 10^{-6}$ & 2.5033 & $1.3038 \times 10^{-5}$ & 3.0195 \\
80 & $1.0698 \times 10^{-3}$ & 4.0338 & $5.0907 \times 10^{-5}$ & 3.7021 & $1.0572 \times 10^{-4}$ & 3.9489 \\
40 & $1.7522 \times 10^{-2}$ & 3.0825 & $6.6257 \times 10^{-4}$ & 3.4844 & $1.6326 \times 10^{-3}$ & 3.4668 \\
20 & $1.4843 \times 10^{-1}$ & - & $7.4154 \times 10^{-3}$ & - & $1.8051 \times 10^{-2}$ & - \\
\hline
\end{tabular}

Table 3: Accuracy test for the isentropic periodic vortex propagation by the simplified scheme. 


\subsection{The Shu-Osher problem}

The above two standard test cases quantitatively prove that the accuracy is not sacrificed despite the simplifications. In the following, two more cases with discontinuities or viscous effect will be considered for comparison between the schemes before and after simplifications, further demonstrating that no accuracy loss is observed for more general flow simulations. We choose one 1-D case and one 2-D case, so that the efficiency improvement can also be investigated with the two cases.

To show the individual effect of the two simplifications made in Section 4 , three methods are compared in this section:

- The original method. This is the method proposed in Ref. [17. It uses the distribution function in Eq. 31] and the coefficients in Eq. 10p.

- The S1 method. This method is the one with Simplification 1 but without Simplification 2. It uses the distribution function in Eq. (31) and the coefficients in Eq. 26.

- The simplified method. This is the scheme with both simplifications. It uses the distribution function in Eq. (34) and the coefficients in Eq. 267.

We ensure that all computations are exactly the same except the evolution model. All computations are run on the same computer. Hence it is reasonable to compare the computational time of different schemes for the same problem to get a quantitative conclusion on the efficiency enhancement.

For the 1-D case we consider the Shu-Osher problem. This problem is a test case for simulating the interaction of a moving shock and a smooth density fluctuation 24. The initial condition is

$$
(\rho, U, p)= \begin{cases}(3.857134,2.629369,10.33333), & -5 \leq x<-4, \\ (1+0.2 \sin (5 x), 0,1), & -4 \leq x \leq 5 .\end{cases}
$$

The CFL number used is 0.6 . The density distributions at $t=1.8$ obtained by the three methods on a grid with 400 cells are compared in Fig. 1. Notice that this is an inviscid case, and no artificial dissipation is added during the computation. So Simplification 2 has no effect since it is a modification related to the viscous terms. Then the results of the S1 method and the simplified method are exactly the same. The results of the original method and the simplified method are also very similar. Both can resolve the high-frequency oscillations very well. Small differences are visible only near the strong discontinuity around $x=2.4$.

The computational efficiency is shown in Table 4 . The CPU time for a complete computation (to the nondimensional time $t=1.8$ ) with 1000 cells is compared. For 1-D cases, the speedup after the simplifications is $267 \%$. 

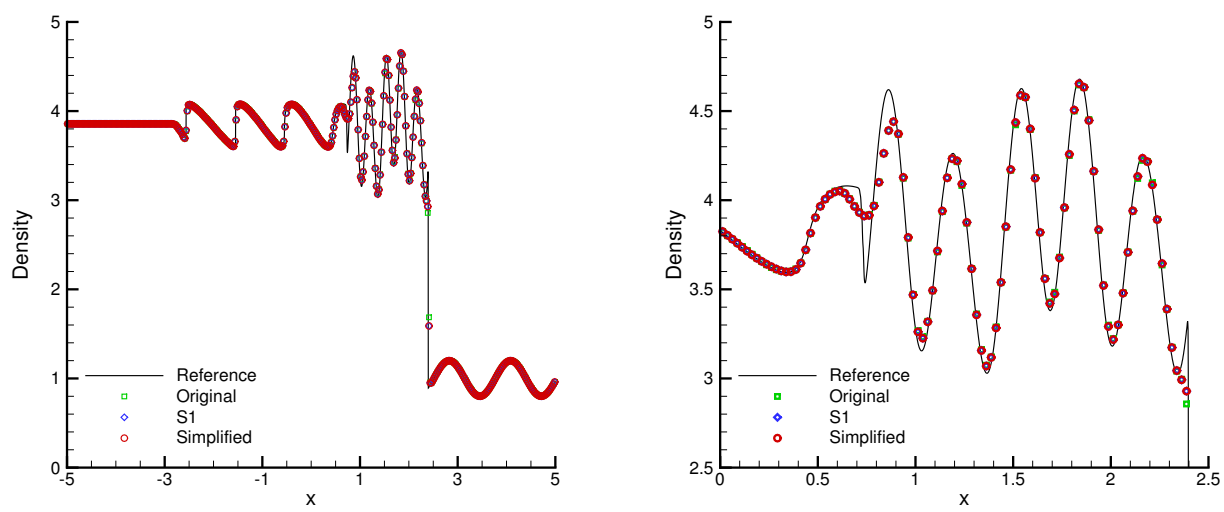

Figure 1: Density distribution at $t=1.8$ of the Shu-Osher problem. 400 cells are used. The reference solution is grid-converged solution obtaind by 10000 cells.

\begin{tabular}{ccc}
\hline Method & CPU time (seconds) & Speedup \\
\hline Original & 13.67 & - \\
S1 & 5.34 & $156 \%$ \\
Simplified & 3.72 & $267 \%$ \\
\hline
\end{tabular}

Table 4: 1-D efficiency evaluation for different methods based on the Shu-Osher problem. The CPU time for a complete computation till $t=1.8$ with 1000 cells is compared.

\subsection{The viscous shock tube problem}

We consider the test case proposed by Daru and Tenaud 25]. A diaphragm is vertically located in the middle of a square 2-D shock tube with unit side length, separating the space into the left and right parts. The initial state in non-dimensional form is given by

$$
(\rho, U, V, p)=\left\{\begin{array}{lc}
(120,0,0,120 / \gamma), & 0 \leq x \leq 0.5, \\
(1.2,0,0,1.2 / \gamma), & 0.5<x \leq 1 .
\end{array}\right.
$$

For air, the specific hear ratio $\gamma=1.4$ and the Prandtl number $\operatorname{Pr}=0.73$. All boundaries of the tube is non-slip and adiabatic. When the diaphragm is broken at $t=0$, a shock forms and moves towards the right, followed by a contact discontinuity. The Mach number of the shock is 2.37. Simultaneously, a rarefaction wave expands in both the left and right directions. After reaching the right wall, the shock is reflected back and moves to the left, interacting with the contact discontinuity and then the rarefaction wave. In viscous cases, a thin boundary layer is generated by the shear between the shock and the horizontal boundaries, resulting in complex shock-wave/boundary-layer interactions. Detailed analyses of the process can be found in Ref. [26]. 
The non-dimensional time for comparison is $t=1$. Only the domain $[0,1] \times[0,0.5]$ is computed due to symmetry of the problem. The CFL number is 1.0. First we consider the case at Re $=200$. A $500 \times 250$ grid is employed. The density distribution by the simplified method is shown in Fig. 22 The height of the primary vortex is compared with those reported in Ref. 27, 28, as listed in Table 5 A good agreement is reached.

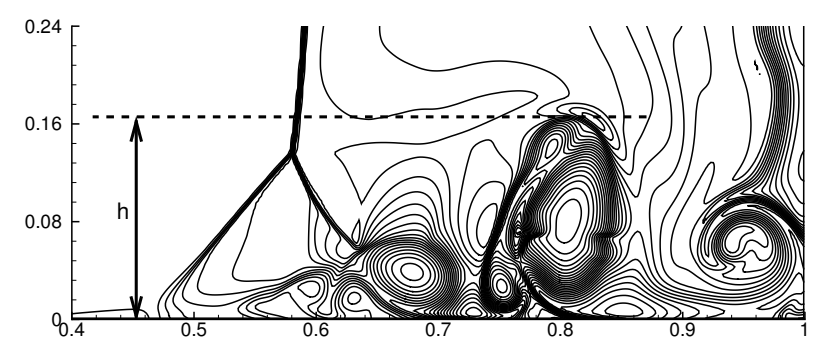

Figure 2: Density distribution at $t=1$ of the viscous shock tube problem at $\operatorname{Re}=200$. The grid used is $500 \times 250$.

\begin{tabular}{ccccc}
\hline Scheme & AUSMPW $+[27]$ & M-AUSMPW $+[27]$ & 4th-order GKS [28] & present \\
\hline Height $(h)$ & 0.163 & 0.166 & 0.171 & 0.166 \\
\hline
\end{tabular}

Table 5: Comparison of the primary vortex height at $R e=200$ for different schemes on uniform $500 \times 250$ grid.

To demonstrate the performance of the simplifications in such a complex viscous problem with a number of discontinuities in the flow field, Fig. 3 compares the results on a $300 \times 150$ grid by the original method and the simplified method. The difference is almost indistinguishable.
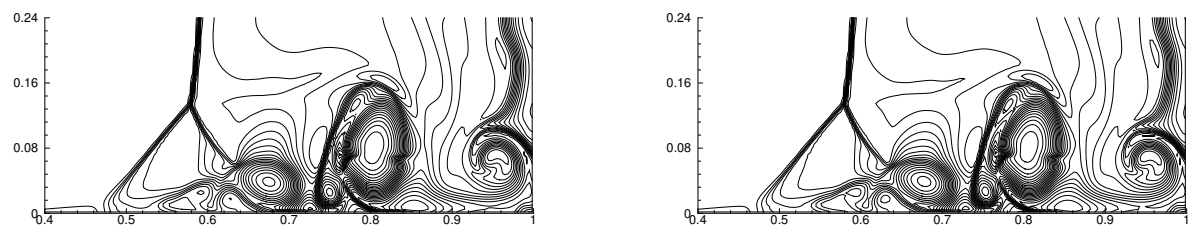

Figure 3: Density distribution at $t=1$ of the viscous shock tube problem at Re $=200$ with a $300 \times 150$ grid. Left: the original method. Right: the simplified method.

For a more accurate pointwise comparison, the density distributions along the bottom wall at $t=1$ are plotted in Fig. The reference solution in the figure is the grid-converged solution on a very fine $(1500 \times 750)$ grid. It is found that some differences exist on the $300 \times 150$ grid. But when the grid is refined to $500 \times 250$, the difference becomes very small. On both grids, the results of the S1 method and the simplified method are very similar, and the results of the simplified method are no worse than those of the original method. In fact, the performance of the simplified method is even slightly better. This phenomenon may be due to 
the fact that by applying Simplification 1, the computations of very high moments of the gas distribution function are avoided, so that less error is introduced during the computation (see Section 4). The original method with many higher-order moment terms may have the dynamical effect corresponding to the Burnett equations, which may not be physically valid [29, 30].
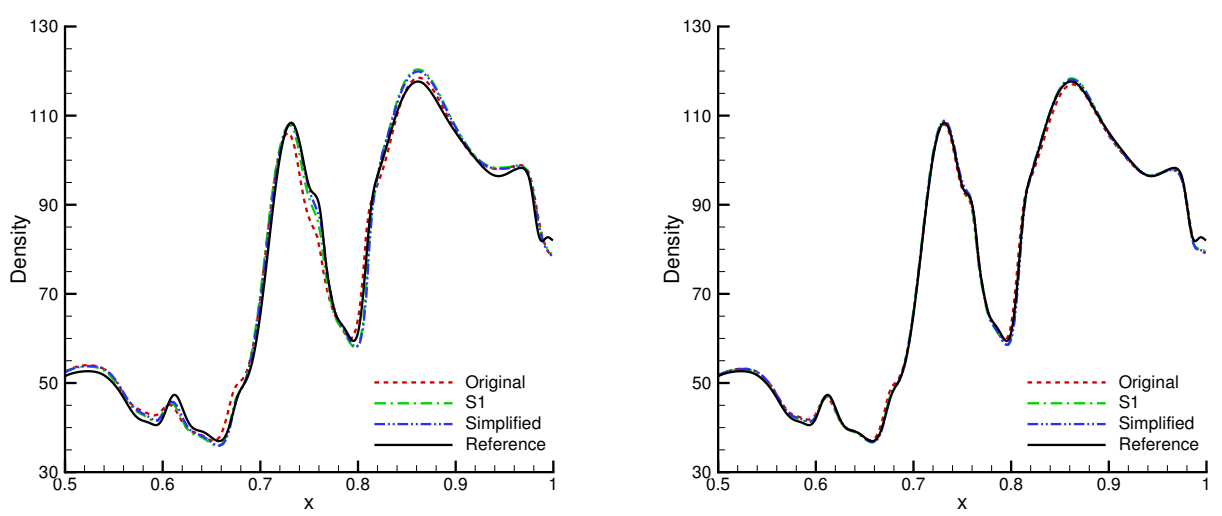

Figure 4: Density distribution along the bottom wall at $t=1$ of the viscous shock tube problem. $\operatorname{Re}=200$. The grid used is $300 \times 150$ (left) and $500 \times 250$ (right). The reference solution is grid-converged solution on a very fine $(1500 \times 750)$ grid

Table 6 shows the efficiency comparison. The CPU time for one time step on a $1000 \times 500$ grid is compared. The simplified method is about 4 times faster than the original one.

\begin{tabular}{ccc}
\hline Method & CPU time (seconds) & Speedup \\
\hline Original & 18.62 & - \\
S1 & 6.68 & $179 \%$ \\
Simplified & 3.76 & $395 \%$ \\
\hline
\end{tabular}

Table 6: 2-D efficiency evaluation for different methods based on the viscous shock tube problem. The CPU time for the computation of one time step on a $1000 \times 500$ grid is compared.

The viscous shock tube problem with a higher Reynolds number $\operatorname{Re}=1000$ is also simulated by the simplified scheme, in which case the flow becomes more complex. Fig. 5 shows the density distribution at $t=1$ on a $2000 \times 1000$ grid. The small-scale structures are clearly presented. The distribution is very much similar to the result in Ref. 26].

The above numerical examples show that the efficiency improvement in 2-D case is larger than that in 1-D case. This is because for the gas-kinetic method, a 2-D scheme is not a simply doubled 1-D scheme. Derivatives in the second dimension participate in the evolution model due to its multidimensionality, and 


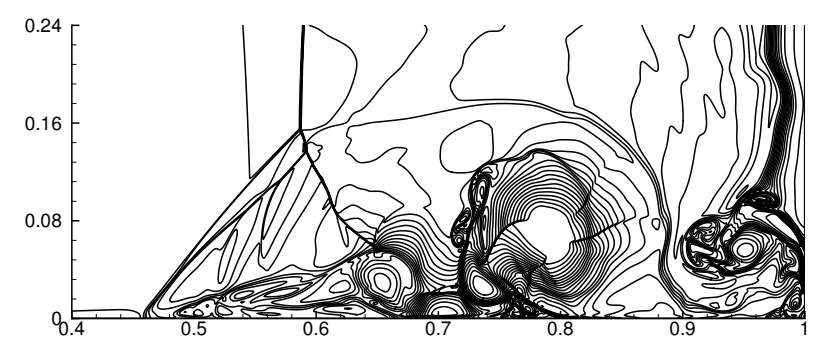

Figure 5: Density distribution at $t=1$ of the viscous shock tube problem at $R e=1000$. The grid used is $2000 \times 1000$.

the cross terms of space- and time-derivatives are also introduced. Similarly, when three spatial dimensions are considered, more terms will appear. Therefore, by using the simplifications in this paper, more gain in computational efficiency is expected for 3-D problems.

\section{Other Computational Results}

Additional test cases are presented in this section to further demonstrate the accuracy, robustness and versatility of the simplified scheme, including inviscid, viscous and nearly incompressible flows and highspeed flows with discontinuities. All the results have good agreement with available theoretical or benchmark solutions by other methods. In the computations, all grids used are uniform with $\Delta x=\Delta y$. The CFL number is set to be 0.6 if not specified.

\subsection{1-D Riemann problem}

Consider the blast wave problem proposed by Woodward and Colella [31]. The initial condition is given by

$$
(\rho, U, p)=\left\{\begin{array}{lc}
(1,0,1000), & 0 \leq x<10, \\
(1,0,0.01), & 10 \leq x<90, \\
(1,0,100), & 90 \leq x \leq 100 .
\end{array}\right.
$$

The density and pressure distributions at $t=3.8$ are plotted in Fig. 6. It is seen that the present result has a comparable behaviour with the original high-order GKS method, whose result is displayed in Ref. [17. 

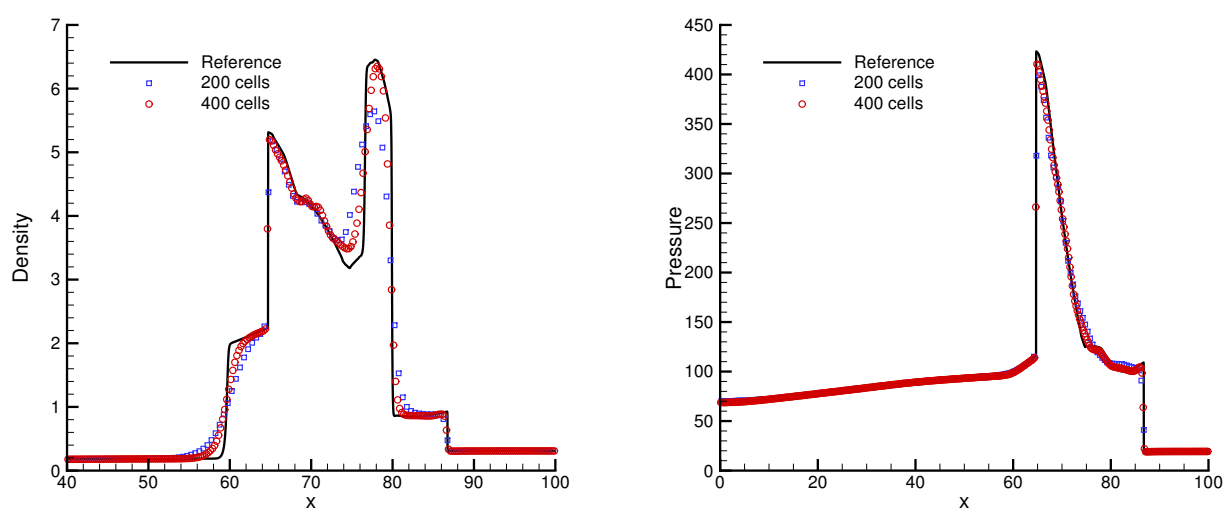

Figure 6: Density (left) and pressure (right) distributions at $t=3.8$ of the blast wave problem. The reference solution is grid-converged solution obtaind with 10000 cells.

\subsection{2-D Riemann problem}

This case is one of the 2-D Riemann problems listed in Ref. [32]. The computational domain is $[0,1] \times[0,1]$. We use the initial conditions given in Ref. [33]:

$$
(\rho, U, V, p)=\left\{\begin{array}{l}
(1.5,0,0,1.5), \quad x \geq 0.8, y \geq 0.8 \\
(0.5323,1.206,0,0.3), \quad x<0.8, y \geq 0.8 \\
(0.138,1.206,1.206,0.029), \quad x<0.8, y<0.8 \\
(0.5323,0,1.206,0.3), \quad x \geq 0.8, y<0.8 .
\end{array}\right.
$$

At $t>0$, four shock waves form at the interfaces of the 4 regions of different initial state. They interact at the junction point and a system of complex structures appear. Two different grids are used, $500 \times 500$ and $1000 \times 1000$. The results at $t=0.8$ are presented. Fig. 7 shows the density distribution. Fig. 8 shows the vorticity magnitude distribution. Good agreements are reached with the results in Ref. [33. The small vortices induced by flow instabilities are well resolved on the $1000 \times 1000$ grid.

\subsection{Laminar boundary layer}

The computational domain is $[-0.2,1] \times[0,0.5]$, a flat plate with the length $L=1$ is located from $x=0$. $360 \times 150$ grid cells are uniformly distributed with $60 \times 150$ cells ahead of the plate. The mean flow Mach number is 0.15 , the Reynolds number based on the plate length is $\operatorname{Re}=U_{\infty} L / \nu=3 \times 10^{4}$. At the lower boundary, the symmetric Euler reflection boundary condition is adopted for $x<0$ and the non-slip condition is used for $x \geq 0$. At the right boundary, simple extrapolation is used. At other boundaries non-reflection boundary conditions based on Riemann invariants are applied. Fig. 9 shows the velocity distribution at 

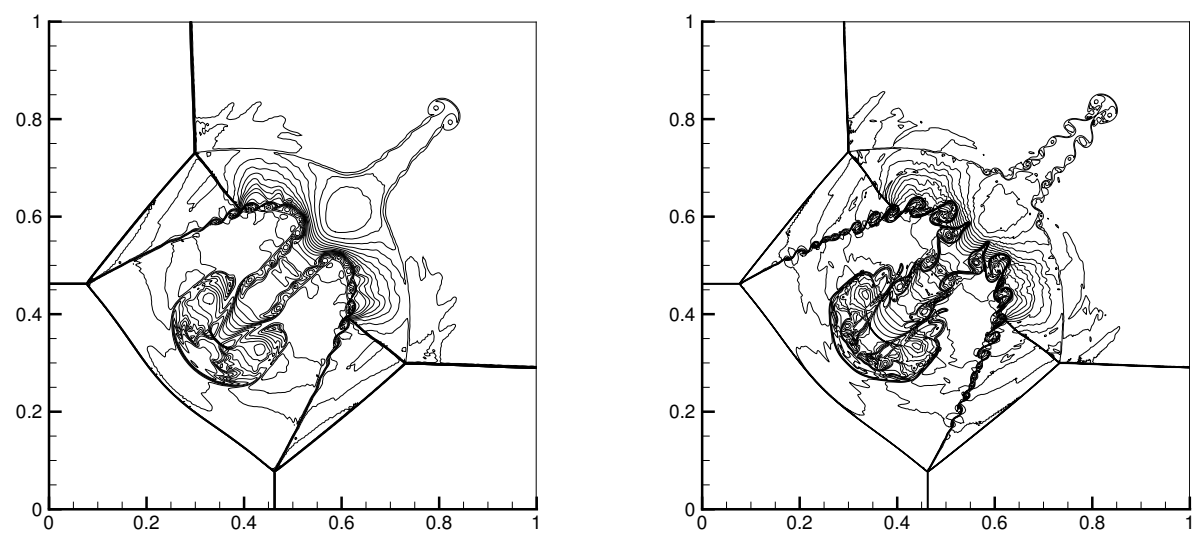

Figure 7: Density distribution at $t=0.8$ of the 2-D Riemann problem. 25 contours equally spaced from 0.2 to 1.7 . $500 \times 500$ grid (left) and $1000 \times 1000$ grid (right).
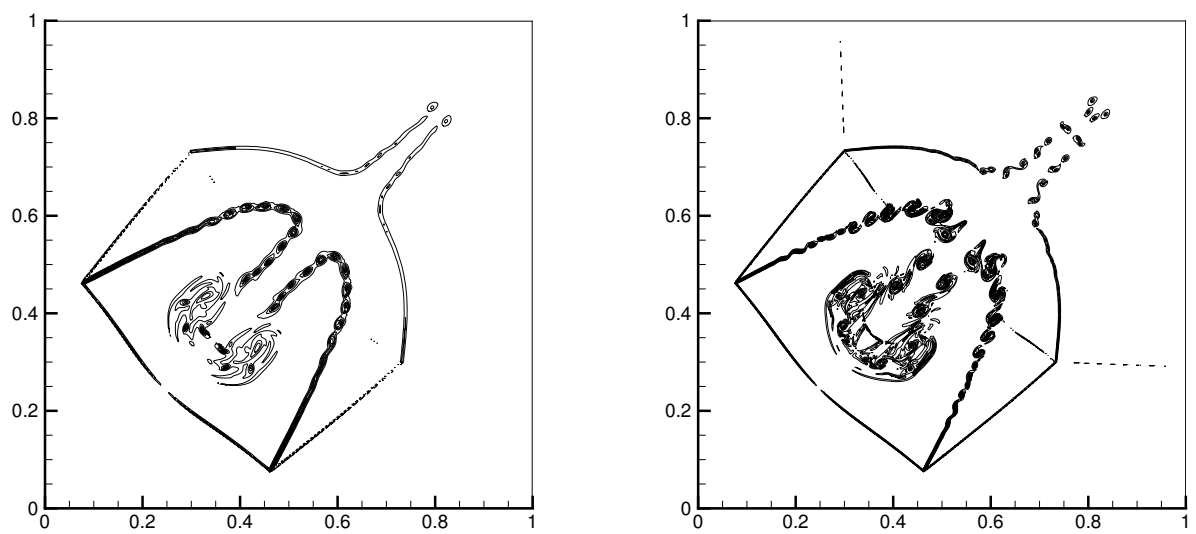

Figure 8: Vorticity magnitude distribution at $t=0.8$ of the 2-D Riemann problem. 21 contours equally spaced from 20 to 600. $500 \times 500$ grid (left) and $1000 \times 1000$ grid (right).

three different locations. The non-dimensional variables are defined as $U^{*}=U / U_{\infty}, V^{*}=V / \sqrt{\nu U_{\infty} / x}$ and $\eta=y / \sqrt{\nu x / U_{\infty}}$. The results of the simplified method agree well with the Blasius solution. The boundary layer is resolved with as few as 5 grid points.

It is noted that in this case, the small vertical velocity is rather sensitive to the reconstruction scheme. Fig. 10 shows the velocity distribution obtained with the 5th-order monotonicity-preserving (MP) reconstruction [34 instead of the WENO-JS reconstruction used to produce the results in Fig. 9 It is clear that the 

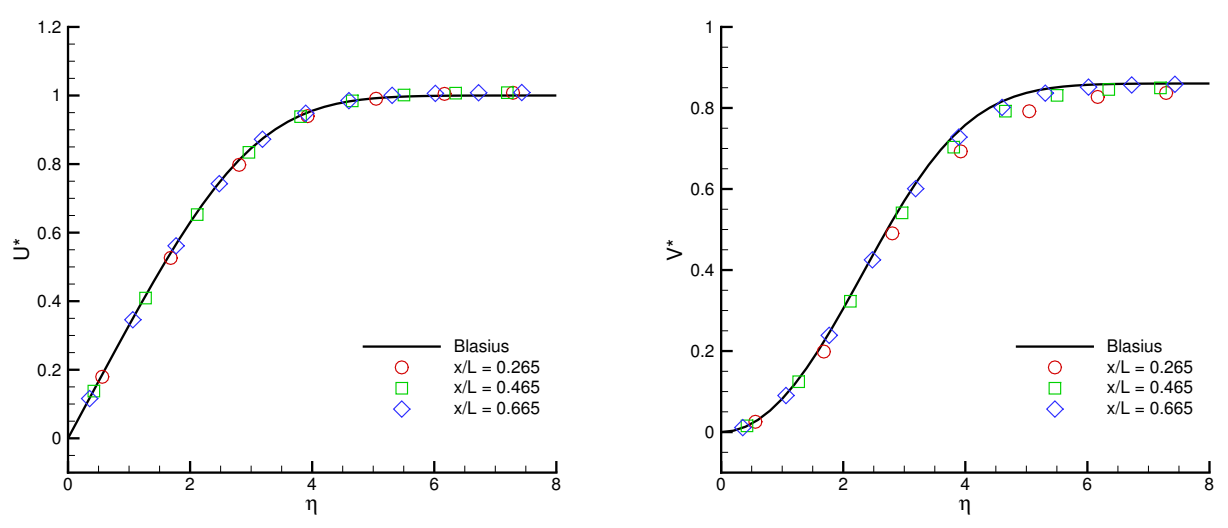

Figure 9: Non-dimensional streamwise (left) and transverse (right) velocitiy distribution of a laminar boundary layer.

MP reconstruction leads to an obviously better result for the transverse velocity distribution. However, for flows with discontinuities such as shocks, the WENO-JS reconstruction is preferred for enhanced robustness.
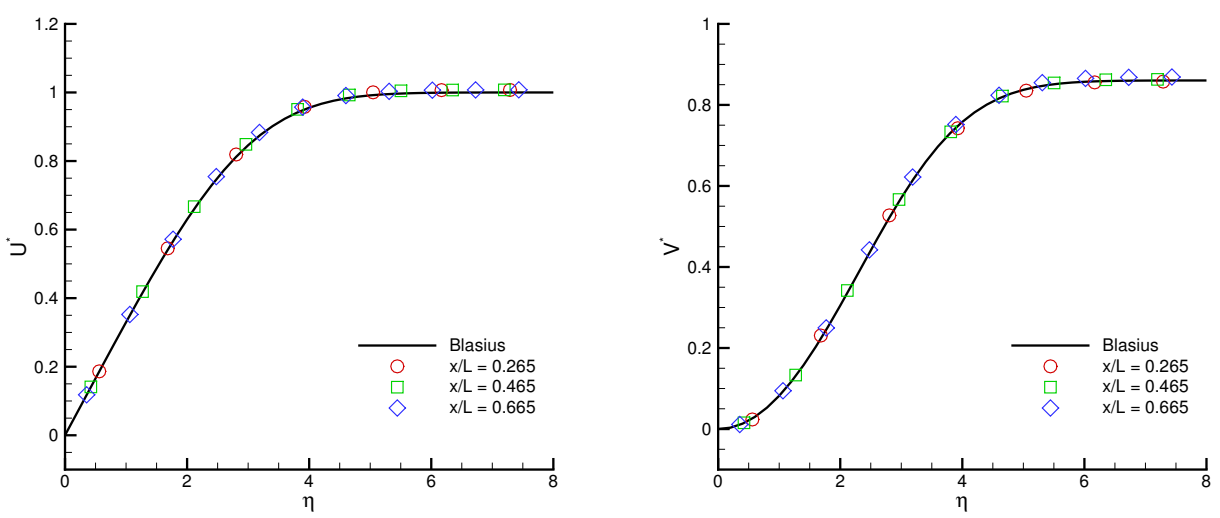

Figure 10: Non-dimensional streamwise (left) and transverse (right) velocitiy distribution of a laminar boundary layer, obtained with a 5th-order monotonicity-preserving reconstruction.

\subsection{Low-speed lid-driven cavity flow}

The fluid is bounded in a cavity with unit side length and driven by the top lid. The lid moves to the right with a uniform velocity. The Mach number is set to be 0.3 , the adiabatic non-slip condition is applied on all boundaries. Since it is a test case for incompressible flow, most simulations in the past use continuous initial reconstruction at the cell interface to minimize the kinematic dissipation [35. However, here we still use the shock capturing WENO reconstruction for consistency. This case is run at $\operatorname{Re}=1000$ and $\operatorname{Re}=3200$. 
For both cases we use a $65 \times 65$ grid. The reference data are from Ref. [36]. The streamlines are presented in Fig. 11. where the vortex structures are clearly seen. Fig. 12 shows the velocity profiles at the central lines of the cavity. The results on the $65 \times 65$ grid match the reference data for Reynolds numbers as high as 3200 very well.
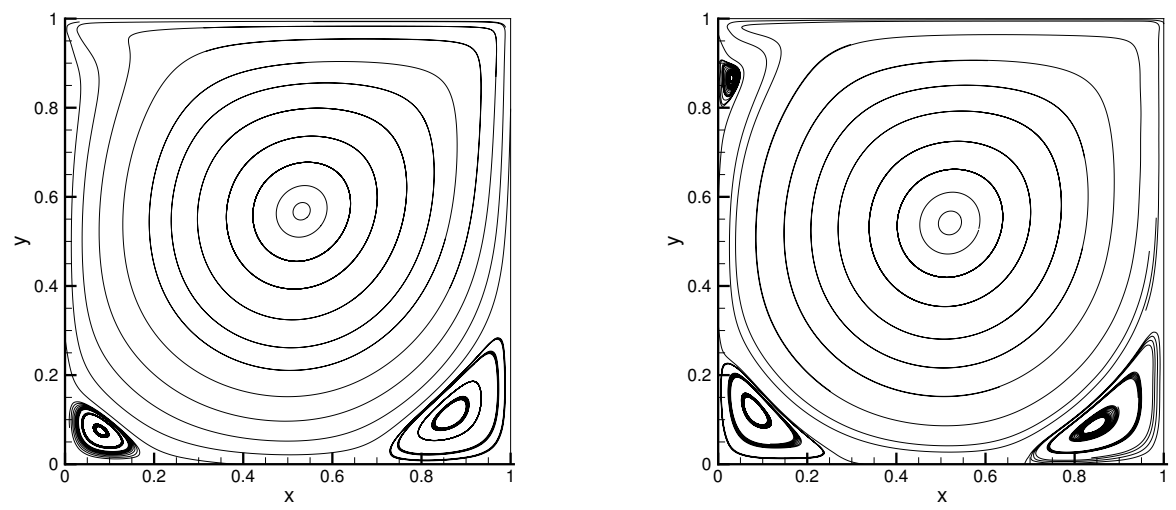

Figure 11: Streamlines of the lid-driven cavity flow with $R e=1000$ (left) and $R e=3200$ (right).

\section{Conclusions}

In this paper, two simplifications have been introduced on the baseline gas evolution model of a high-order gas-kinetic scheme with WENO reconstruction. The modifications are designed based on the physical analysis of the high-order terms in the time-evolving gas distribution function in order to improve computational efficiency while preserving accuracy. The resultant method significantly reduces the computational cost. The method preserves an overall 3rd-order accuracy in both space and time. It is about 4 times more efficient than the original method for 2-D cases, with even better potential for 3-D applications. A wide range of cases involving inviscid, viscous, low-speed and high-speed flow with and without discontinuities are tested to show the robustness, accuracy, and efficiency of the simplified scheme. The simplifications and the design of the artificial dissipation introduced in this paper can also be adopted by other kinds of high-order gas-kinetic methods.

\section{Acknowledgements}

The current work of K. Xu is supported by Hong Kong Research Grant Council (620813, 16211014, 16207715), and National Science Foundation of China (91330203,91530319). 

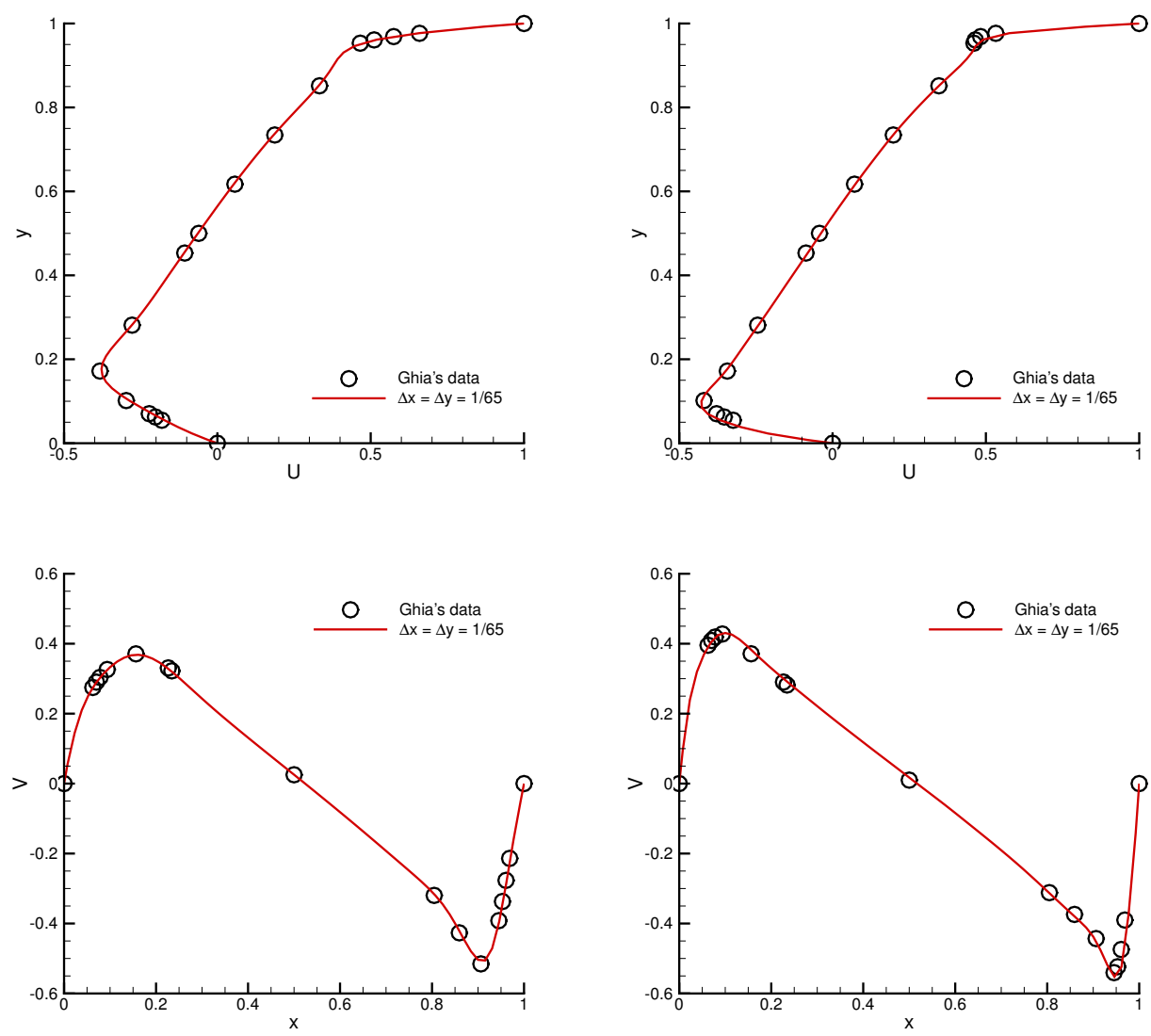

Figure 12: Velocity profile of the cavity flow with $\mathrm{Re}=1000$ (left) and $\mathrm{Re}=3200$ (right). $U$ velocity is taken at the line $x=0.5$, and $V$ velocity is taken at the line $y=0.5$.

\section{References}

[1] K. Xu, Gas-kinetic schemes for unsteady compressible flow simulations, von Karman Institute report (1998).

[2] K. Xu, A gas-kinetic BGK scheme for the Navier-Stokes equations and its connection with artificial dissipation and Godunov method, Journal of Computational Physics 171 (2001) 289-335.

[3] A. Harten, B. Engquist, S. Osher, S. R. Chakravarthy, Uniformly high order accurate essentially nonoscillatory schemes III, Journal of Computational Physics 71 (1987) 231-303.

[4] X.-D. Liu, S. Osher, T. Chan, Weighted essentially non-oscillatory schemes, Journal of Computational Physics 115 (1994) 200-212. 
[5] W. H. Reed, T. R. Hill, Triangular mesh methods for the neutron transport equation, Technical Report LA-UR-73-479, Los Alamos Scientific Laboratory, 1973.

[6] B. Cockburn, C.-W. Shu, TVB Runge-Kutta local projection discontinuous Galerkin finite element method for conservation laws II: general framework, Mathematics of Computation 52 (1989) 411-435.

[7] B. Cockburn, C.-W. Shu, Nonlinearly stable compact schemes for shock calculations, SIAM Journal on Numerical Analysis 31 (1994) 607-627.

[8] X. Deng, H. Maekawa, Compact high-order accurate nonlinear schemes, Journal of Computational Physics 130 (1997) 77-91.

[9] Z. J. Wang, Spectral (finite) volume method for conservation laws on unstructured grids: basic formulation, Journal of Computational Physics 178 (2002) 210-251.

[10] Y. Liu, M. Vinokur, Z. J. Wang, Spectral difference method for unstructured grids I: basic formulation, Journal of Computational Physics 216 (2006) 780-801.

[11] V. A. Titarev, E. F. Toro, Finite-volume WENO schemes for three-dimensional conservation laws, Journal of Computational Physics 201 (2004) 238-260.

[12] M. Ben-Artzi, J. Falcovitz, A second-order Godunov-type scheme for compressible fluid dynamics, Journal of Computational Physics 55 (1984) 1-32.

[13] M. Ben-Artzi, J. Li, G. Warnecke, A direct Eulerian GRP scheme for compressible fluid flows, Journal of Computational Physics 218 (2006) 19-43.

[14] V. A. Titarev, E. F. Toro, ADER: Arbitrary high order Godunov approach, Journal of Scientific Computing 17 (2002) 609-618.

[15] Q. Li, K. Xu, S. Fu, A high-order gas-kinetic Navier-Stokes flow solver, Journal of Computational Physics 229 (2010) 6715-6731.

[16] G. Kumar, S. S. Girimaji, J. Kerimo, WENO-enhanced gas-kinetic scheme for direct simulations of compressible transition and turbulence, Journal of Computational Physics 234 (2013) 499-523.

[17] J. Luo, K. Xu, A high-order multidimensional gas-kinetic scheme for hydrodynamic equations, Science China Technological Sciences 56 (2013) 2370-2384.

[18] N. Liu, H. Tang, A high-order accurate gas-kinetic scheme for one- and two-dimensional flow simulation, Communications in Computational Physics 15 (2014) 911-943. 
[19] J. Luo, L. Xuan, K. Xu, Comparison of fifth-order WENO scheme and finite volume WENO-gas-kinetic scheme for inviscid and viscous flow simulation, Communications in Computational Physics 14 (2013) 599-620.

[20] P. L. Bhatnagar, E. P. Gross, M. Krook, A model for collision processes in gases. I. Small amplitude processes in charged and neutral one-component systems, Physical Review 94 (1954) 511-525.

[21] G.-S. Jiang, C.-W. Shu, Efficient implementation of weighted ENO schemes, Journal of Computational Physics 126 (1996) 202-228.

[22] C.-W. Shu, Essentially non-oscillatory and weighted essentially non-oscillatory schemes for hyperbolic conservation laws, Institute for Computer Applications in Science and Engineering (1997).

[23] T. Ohwada, K. Xu, The kinetic scheme for the full-Burnett equations, Journal of Computational Physics 201 (2004) 315-332.

[24] C.-W. Shu, S. Osher, Efficient implementation of essentially non-oscillatory shock-capturing schemes II, Journal of Computational Physics 83 (1989) 32-78.

[25] V. Daru, C. Tenaud, Evaluation of TVD high resolution schemes for unsteady viscous shocked flows, Computers \& Fluids 30 (2001) 89-113.

[26] V. Daru, C. Tenaud, Numerical simulation of the viscous shock tube problem by using a high resolution monotonicity-preserving scheme, Computers \& Fluids 38 (2009) 664-676.

[27] K. H. Kim, C. Kim, Accurate, efficient and monotonic numerical methods for multi-dimensional compressible flows: Part I: Spatial discretization, Journal of Computational Physics 208 (2005) 527-569.

[28] L. Pan, K. Xu, Q. Li, J. Li, An efficient and accurate two-stage fourth-order gas-kinetic scheme for the Euler and Navier-Stokes equations, Journal of Computational Physics 326 (2016) 197-221.

[29] X. Zhong, R. W. MacCormack, D. R. Chapman, Stabilization of the Burnett equations and application to hypersonic flows, AIAA Journal 31 (1993) 1036-1043.

[30] K. Xu, Regularization of the Chapman-Enskog expansion and its description of shock structure, Physics of Fluids 14 (2002) 17-20.

[31] P. Woodward, P. Colella, The numerical simulation of two-dimensional fluid flow with strong shocks, Journal of Computational Physics 54 (1984) 115-173. 
[32] T. Zhang, Y. Zheng, Conjecture on the structure of solutions of the Riemann problem for two-dimensional gas dynamics systems, SIAM Journal on Mathematical Analysis 21 (1990) 593-630.

[33] L. Pan, J. Li, K. Xu, A few benchmark test cases for higher-order Euler solvers, arXiv:1609.04491v1 [math.NA], 15 Sept. 2016.

[34] A. Suresh, H. T. Huynh, Accurate monotonicity-preserving schemes with Runge-Kutta time stepping, Journal of Computational Physics 136 (1997) 83-99.

[35] K. Xu, X. He, Lattice Boltzmann method and gas-kinetic BGK scheme in the low-Mach number viscous flow simulations, Journal of Computational Physics 190 (2003) 100-117.

[36] U. Ghia, K. N. Ghia, C. T. Shin, High-Re solutions for incompressible flow using the Navier-Stokes equations and a multigrid method, Journal of Computational Physics 48 (1982) 387-411. 